\title{
The balance between the serum levels of IL- 6 and IL-10 cytokines discriminates mild and severe acute pneumonia
}

Rita de Cássia Coelho Moraes de Brito ${ }^{*^{*}}$, Norma Lucena-Silva ${ }^{2}$ D, Leuridan Cavalcante Torres ${ }^{3}$, Carlos Feitosa Luna ${ }^{4}$, Jaílson de Barros Correia ${ }^{3}$ and Giselia Alves Pontes da Silva ${ }^{5}$

\begin{abstract}
Background: To identify markers for earlier diagnosis of severe pneumonia, we assess the correlation between serum cytokine profile of children with different pneumonia severity.

Methods: In 25 hospitalized children, 7 with mild pneumonia and 18 with severe pneumonia, the serum concentration of 11 cytokines in three sampling times were dosed. Statistical analysis included parametric and non-parametric tests, Pearson correlation and ROC curve for cut-off definition of cytokines.

Results: At admission, IL-6 serum levels were high in mild or severe pneumonia, and was associated to vomiting $(P=0$. 019) in both groups; and also to dyspnea $(P=0.012)$ and white blood cell count $(P=0.045)$ in patients with severe pneumonia. IL-10 levels were also high in patients with pneumonia and were associated to lymphocytosis $(P=0.025)$. The ROC curve of the IL-6:IL-10 serum levels ratio discriminated severe pneumonia cases at admission, and persistence of infection in the third day of antibiotic therapy, with positive predictive values of $93 \%$ and $89 \%$, respectively.
\end{abstract}

Conclusions: The balance between IL-6 and IL-10 serum levels showed to be a more discriminative marker for severity definition and evaluation of recovery in patients with pneumonia.

Keywords: Pneumonia, Inflammation, Cytokines, Immune response

\section{Background}

Community acquired pneumonia (CAP) is a serious illness of the lower respiratory tract, responsible for high morbidity in children, especially those younger than five years old [1-3]. The World Health Organization (WHO) uses clinical manifestations as a parameter for definition of severity ratings (pneumonia and severe pneumonia) [4]. The gradient of clinical severity arises from a complex interaction between environmental factors, pathogenicity of the etiologic agent and host characteristics, including immunological competence, malnutrition, anemia and other comorbidities [5-13]. Clinical data associated to blood count and serum level of the Creactive protein $(\mathrm{CRP})$ are determinants for therapeutic

\footnotetext{
* Correspondence: moraesdebrito@gmail.com

${ }^{1}$ Institute of Integral Medicine Professor Fernando, Figueira (IMIP), Pediatrics, Rua Dona Benvinda de Farias 159, apt 1101, Boa Viagem, Recife,

Pernambuco, Brazil

Full list of author information is available at the end of the article
}

management of acute respiratory disease in clinical practice [3, 14-16]. Blood count and serum levels of CRP are low-cost laboratory tests, accessible in health services in countries with limited resources and their results help the differentiation between viral or bacterial respiratory infection [3, 14, 17]. High levels of CRP are caused bythe activity of circulating tumor necrosis factor (TNF) in the liver as a response to the infectious process; therefore, CRP is considered a nonspecific marker [18].

Recently, serum and bronchoalveolar levels of cytokines have been related to severity of pulmonary inflammatory process $[9,19-21]$. During the early stages of pneumonia, the alveolar macrophages produce a variety of cytokines and inflammatory chemokines, which attract and activate polymorphonuclear leukocytes that will mount an appropriate immune response in the lung parenchyma [22-24]. Circulating levels of TNF, Interleukin (IL)-1, IL-6, IL-8, IL-12 and interferon-gamma (IFN$\gamma)$ are found in patients with CAP $[7,9,19-21]$. The 
cytokines IL-1, TNF and IL-6 are important for the acute phase response $[7,9,25]$, while modulation of inflammatory responses of the airways is related to the expression of the anti-inflammatory cytokines,IL-10 and IL-4 $[6,8,24]$. Studies on the inflammatory process during acute respiratory disease have reported conflicting results. Most studies that seek to relate the severity of acute respiratory disease with cytokines serum levels have evaluated cytokine levels in a single time point and samples were collected from adult patients, who may have different inflammatory responses from those observed in children due to the anatomical-functional differences of the pulmonary alveoli, in addition to the immune system immaturity $[7,9-13,21,26]$. The aim of the present study was to evaluate the correlation between the cytokines profile in the serum of children and adolescents with different pneumonia severity, defined by clinical manifestations and alterations in the blood count, in order to identify markers for early diagnosis of severe pneumonia.

\section{Methods}

\section{Design and the subjects of the study}

This was an observational, prospective and exploratory study, which investigated the association between serum cytokines levels with pneumonia severity. The classification of clinical severity was based on the criteria defined by WHO and established in the Brazilian Guidelines for CAP in Pediatrics [3]. Briefly, cough or tachypnea (respiratory rate $\geq 50$ breaths per minute in an infant or $\geq$ 40 breaths per minute in one year or older children) with radiological evidence of pulmonary consolidation was defined as pneumonia. We considered severe pneumonia either the presence of chest indrawing in children with cough or difficult breathing, which were criteria for severe pneumonia by $\mathrm{WHO}$, or presence of severe respiratory distress or inability to drink or central cyanosis in a child with cough or difficult breathing, which are criteria for classification of a very severe pneumonia by WHO. Cytokine levels of children with pneumonia were compared with cytokine levels of children with severe pneumonia.

The patients evaluated in the present study were admitted to three hospitals (Hospital da Restauração, Hospital Helena Moura and the Instituto de Medicina Integral Professor Fernando Figueira-IMIP) in the municipality of Recife, northeastern Brazil, from June to December 2012. All three hospitals belong to the network of public health assistance of the Brazilian Health System (Sistema Único de Saúde-SUS).

The exclusion criteria were age under six months, previous history of immunodeficiency, clinical suspicion of tuberculosis, heart disease, neurological disease, cystic fibrosis, bronchiolitis obliterans and severe persistent asthma. Children that were transferred from other hospitals, and were using antibiotics therapy for more than 3 days at the admission were also excluded, because we were not able to collect biological samples for analysis in two of three time points defined in the research protocol.

The present study was approved by the Research Ethics Committees of the IMIP hospital (protocol 2886) and the Hospital da Restauração (protocol CAAE-0014.0.102.00011). A clearance letter was also issued by the Health Secretary of the state of Pernambuco authorizing the research implementation in the Hospital Helena Moura. Data collection was performed after obtaining written Informed Consent signed by the children's legal guardian.

\section{Definition of variables}

A form containing demographic (age, gender, place of residence, socio-economic conditions) and clinical questions (duration of the disease, general symptoms and related acute respiratory disease, including fever, coughing, difficult breathing, subcostal recession, cyanosis and nasal flaring, respiratory frequency, heart rate and oxygen saturation) was filled out at hospital admission for each patient. Presence of complications, such as pleural effusion, respiratory insufficiency and anemia were also investigated. Hemoglobin levels lower than 11,11.5 or $12 \mathrm{~g} / \mathrm{dL}$ defined anemia for children less than five years old, children 5 to 11 years old and adolescents 12 to 14 years old, respectively [27]. Oxygen saturation and heart rate were evaluated in patients with no oxygen supply using the Rossmax Pulse Oximeter SB100. Respiratory frequency was defined as the number of breaths per minute.

Chest $\mathrm{x}$-ray and blood tests were performed at hospital admission. Blood samples were collected for cytokine dosage in three sampling times: on the day of admission (D0) before any medical intervention; three days after the therapeutic scheme had started (D3), when a clinical improvement of $80 \%$ in children treated with proper antibiotics is expected; and eight days (D8) after the therapeutic scheme had started, when complete illness resolution is expected.

\section{Cytokines analysis}

Peripheral blood samples were collected and sent to the laboratory within $40 \mathrm{~min}$. The cytokines IL-8, IL-1 $\beta$, IL6, IL-10, TNF, IL-12p70 and IL-17A, IL-2, IL-4, IFN- $\gamma$ were evaluated by the $\mathrm{BD}^{\mathrm{m}}$ Cytometric Bead Array $\mathrm{Hu}-$ man Inflammation reagents and the Human Th1/Th2/ Th17 Cytokine kit. These tests were chosen because they allow the quantification of several cytokines simultaneously using a small sample volume. The analysis of IL-5 expression was performed by immunoassay using the BD OptEIA ${ }^{\circ}$ Human IL-5 reagent, as recommended by the manufacturer (Becton Dickinson, Franklin Lakes, New Jersey). Of 25 children, cytokines serum levels were determined in 23 at admission, and 17 on D3 and D8 of treatment. 


\section{Data analysis and statistics}

Numerical variables, including age, respiratory and heart frequency and oxygen saturation among others, were subjected to the normality test of Kolmogorov-Smirnov, and those that did not present normal distribution were expressed by median, minimum and maximum values. The median difference was assessed by Mann-Whitney $\mathrm{U}$ non-parametric test. Categorical data were analyzed using absolute and relative frequency tables and comparative analysis of the two studied groups (pneumonia and severe pneumonia) was performed by the Chisquare test or the Fisher's 2-tailed test,when indicated. The non-parametric Friedman test with its respective post-hoc was used for intra-group analysis. A significance level of $5 \%$ was considered in all analyzes.

The correlation between cytokine levels, clinical variables and blood count were evaluated by Pearson correlation coefficient, except for the subcostal recession variable, which was analyzed by the Spearman's coefficient. The ROC curve, area under the curve, sensitivity, specificity, positive and negative predictive value were used to find the cut-offs for the IL6 and IL6:IL10 ratio levels. The statistical analyzes and graphics were performed using the Action 26 Excel software (Microsoft, Albuquerque, New Mexico) and GraphPad Software Inc. (La Jolla, California).

\section{Results}

\section{Patient characterization}

Of the 25 children evaluated in the present study, 7 were diagnosed with pneumonia and 18 with severe pneumonia. Almost all patients had an updated and complete vaccination scheme $(92 \%)$, were breast fed $(88 \%)$ and had adequate weight for age (96\%). Three patients (12\%) lived with more than seven people in the same household, most patients $(80 \%)$ were from the countryside of the state of Pernambuco, and a third (32\%) of the respondents reported the presence of smokers at home. Low maternal schooling was common among the children's legal guardians (96\%).

Severe pneumonia was more frequent among children younger than 5 years old $(P=0.027)$, and there was no difference in disease severity between gender $(P=0.656)$. Coughing and fever were observed in all patients of both groups. However, the duration of fever $(P=0.000)$ and anorexia $(P=0.023)$ during hospitalization were longer in severe patients. The most frequent symptoms in patients with severe pneumonia were subcostal recession $(P<0.000)$, difficult breathing $(P=0.007), \mathrm{O}_{2}$ saturation $(P=0.011)$, wheezing $(P=0.032)$ and vomiting $(P=0.021)$ (Additional file 1: Table S1).

Anemia occurred in $61.1 \%$ of the patients with severe pneumonia and leukocytosis was observed in both studied groups. The median leukocyte count was higher in the pneumonia group than in children with severe pneumonia, but with no statistical difference between the two groups. Patients with severe pneumonia were diagnosed later (median of 10 days) than children with pneumonia (median of 7 days) $(P=0.023)$.

Of the 18 patients with severe pneumonia, 11 (61\%) needed chest drainage for a median of 11 days (range 4 to 29 days). Biochemical analysis and pleural fluid culture were not performed in any of the cases. In nine patients with severe pneumonia (50\%), oxygen was supplied by a Venturi mask for a median of 7 days.

\section{Cytokine profile of patients with pneumonia and severe pneumonia}

Serum circulating levels of TNF, IL-1 $\beta$, IL-8, IL-17 and IL-5 in the three sampling times were similar for both studied groups. At diagnosis, IL-10 levels were lower (3.68 vesus $1.99, P=0.074$ ), and IL-6 levels were higher (44.07 versus $19.98, P=0.155$ ) in children with severe pneumonia than in children with non-severe pneumonia; however, no statistical difference was observed between the two groups. Furthermore, IL-6 serum levels significantly reduced in patients with pneumonia $(P=0.041)$ and severe pneumonia $(P=0.001)$ during recovery (Table 1 ).

There was a correlation between some cytokines and the clinical signs of severity and nonspecific symptoms related to the infection. In patients with severe pneumonia and pneumonia, IL-6 levels were associated with vomiting; however, in the severe pneumonia group, IL-6 levels were also associated to dyspnea, suggesting that this cytokine plays a role in pneumonia severity (Table 2 ).

In patients with pneumonia at hospital admission, IL-10 was associated to lymphocytosis $(P=0.025)$, and in patients with severe pneumonia, the proliferation of defense cells was demonstrated by the positive association between IL-6 levels and leukocyte count $(P=0.045)$ and the levels of TNF $(P=0.025)$ and IL-1 $\beta(P=0.040)$ with monocytosis (Table 2).

\section{Pneumonia recovery and cytokines profile}

Oxygen saturation, temperature, respiratory and cardiac frequencies are predictive paramenters for pneumonia severity, and were evaluated at admission, on D3 and D8 of hospitalization for all children (Additional file 2: Table S2).

At admission, $\mathrm{O}_{2}$ saturation was the most important criterion of severity ( $94 \%$ for severe pneumonia versus $98 \%$ for non-severe pneumonia, $P=0.011$ ), but no significant differences were found in temperature and respiratory or cardiac frequencies. On day D3 of hospitalization, besides the $\mathrm{O}_{2}$ saturation difference $(P=0.021)$, it was possible to distinguish severe pneumonia from non-severe cases based on respiratory frequency $(P=0.030)$ and persistence of fever $(P=0.000)$. On D8 of hospitalization, $\mathrm{O}_{2}$ 
Table 1 Cytokine profiles of patients with pneumonia according to severity and disease duration

\begin{tabular}{|c|c|c|c|c|c|c|c|c|c|}
\hline \multirow[b]{2}{*}{ Cytokines (days) } & \multicolumn{4}{|c|}{ Severe pneumonia } & \multicolumn{4}{|c|}{ Non-severe pneumonia } & \multirow{2}{*}{$\begin{array}{l}\text { Severe } x \text { non-severe pneumonia } \\
{ }^{* * *} \text {-value }\end{array}$} \\
\hline & $\mathrm{Q}_{1}$ & $\mathrm{Q}_{3}$ & Median & ${ }^{*} P$-value & $\mathrm{Q}_{1}$ & $\mathrm{Q}_{3}$ & Median & ${ }^{* *} P$-value & \\
\hline IL10 (D0) & 1.50 & 2.68 & 1.99 & & 2.44 & 4.05 & 3.68 & & 0.074 \\
\hline IL10 (D3) & 1.35 & 3.59 & 1.92 & & 1.41 & 6.12 & 2.31 & & 0.441 \\
\hline IL10 (D8) & 1.99 & 5.55 & 2.59 & 0.417 & 1.72 & 11.43 & 2.16 & 0.549 & 1.000 \\
\hline IL6 (D0) & 26.62 & 108.47 & 44.07 & & 13.55 & 445.55 & 19.98 & & 0.155 \\
\hline IL6 (D3) & 9.27 & 40.48 & 16.45 & & 4.95 & 56.34 & 11.13 & & 0.959 \\
\hline IL6 (D8) & 3.27 & 15.32 & 8.41 & 0.001 & 2.98 & 12.66 & 4.94 & 0.041 & 0.442 \\
\hline
\end{tabular}

Note

* $P$-value is related to the difference in serum cytokines levels in D0, D3 and D8 for children with severe pneumonia

**P-value is related to the difference in serum cytokines levels in DO, D3 and D8 for children with non-severe pneumonia (intragroup analysis considered children with all three samples evaluated)

***P-value is related to comparisons of serum cytokine levels between group of children with severe and non-severe pneumonia in each time point D0, D3 or D8 (Intergroup analtsis considered all samples collected for each time point). Q1, first quartil, and Q3, thrird quartil. $P$-value $\leq 0.05$ was considered significant, significant values are shown in bold

saturation and persistence of fever were not predictive parameters for pneumonia severity, but high respiratory frequency still predicted disease severity $(P=0.008)$.

Considering data of children who had all three samples (D0, D3 and D8) collected, we performed a longitudial analysis for temporal evaluation of the clinical signs recovery. Children with non-severe pneumonia had significant temperature improvement on D3 $(P=$ 0.002). On the other hand, in patients with severe pneumonia, no statistical difference was observed from D0 to D3 $(P=0.393)$, but a significant temperature change occurred from D3 to D8 $(P=0.001)$. The improvement of the respiratory frequency was also faster in patients with non-severe pneumonia than in patients with severe pneumonia. In non-severe pneumonia group, a significant difference of the respiratory frequency occurred from D0 to D3 $(P=0.012)$, while in patients with severe pneumonia, the difference was only noticeable on D8 in comparison with D3 $(P=0.014)$ or D0 $(P=0.001)$ (Additional file 2: Table 2).

Regarding the cytokine levels, the reduction of IL-6 serum levels during recovery of children with severe pneumonia was the effect more pronuanced (Fig. 1). The ratio between the median of IL- 6 and IL-10 serum levels in patients with severe and non-severe pneumonia was respectively 22 and 5 at admission, but 9 and 5 on D3, and 3 and 2 on D8 of antibiotics therapy.

According to the ROC curve analysis, at hospital admission, IL-6 serum levels equal or higher than $21.1 \mathrm{pg} /$ $\mathrm{mL}$ and IL-6:IL-10 ratio of at least 9.61 may discriminate severe pneumonia from mild disease with a sensitivity of $76.5 \%$, corresponding to a positive predictive value of 93\% (Table 3). Furthermore, in the third day of antibiotics therapy, IL6:IL10 serum levels higher than 5.0 predict persistence of the symptoms and may help medical decision in adjusting the therapeutics for severe cases, and discharge mild pneumonia cases. The decrease in the IL-6:IL-10 ratio to 3 in patients with severe pneumonia on D8 coincided with the temperature and respiratory frequency recovery to normal levels.

\section{Discussion}

Pneumonia is a disease associated to poverty conditions related to the environment, the individual, the infectious agent and the healthcare services [3, 4, 28-30]. In the present study, we adopted the criteria published in the Brazilian guidelines for community acquired pneumonia in pediatrics, which follows the former guidelines of WHO [3], allowing that patients $(5-10 \%$ of the cases) with hidden pneumonia, which is characterized by fever and coughing, with or without radiographic changes, and improvement after empirical treatment, may be diagnosed at an early stage $[1,31,32]$. Thus, patients with fever, coughing, tachypnea and radiological changes were diagnosed with pneumonia, and those with subcostal recession were classified with severe pneumonia. In our casuistic, factors such age, anorexia, sibilance, difficult breathing, and vomiting were statistically correlated with pneumonia severity what corroborates with other studies [30, 33-36]. Presence of abdominal pain in children with pneumonia was observed by us as reported by other authors, but without any association with the disease severity [34, 37]. Anemia has a high prevalence in our midst, as demonstrated by Carvalho et al., who found a prevalence of $92 \%$ of healthy children in day care centers in the public health network with hemoglobin levels < $11.0 \mathrm{~g} / \mathrm{dL}$; but it was not also associated to severe pneumonia [12, 38]. The elapsed time between the beginning of the disease and its diagnostic, which can be related to the difficult access to the health services, was associated with disease severity, corroborating with other studies carried out in Brazil and Africa [33, 39]. Coincidently, all children under 5 years old were from countryside and presented severe pnuemonia. In this case, two aspects 
Table 2 Correlation between clinical signs and blood count with cytokines serum levels of patients with severe and non-severe pneumonia

\begin{tabular}{|c|c|c|c|c|c|c|c|c|c|c|c|c|c|c|c|c|c|c|c|c|}
\hline & \multicolumn{2}{|c|}{$\underline{\mathrm{IL}-12 \mathrm{p} 70}$} & \multicolumn{2}{|l|}{ TNF } & \multicolumn{2}{|l|}{$\underline{\mathrm{IL}-10}$} & \multicolumn{2}{|l|}{$\underline{\mathrm{IL}-6}$} & \multicolumn{2}{|l|}{$\underline{\mathrm{LL} 1 \beta}$} & \multicolumn{2}{|l|}{$\underline{\mathrm{IL}-8}$} & \multicolumn{2}{|l|}{ IFN } & \multicolumn{2}{|l|}{ IL-17A } & \multicolumn{2}{|l|}{$\underline{\mathrm{IL} 5}$} & \multicolumn{2}{|c|}{$\underline{\mathrm{IL}-6 / \mathrm{IL}-10}$} \\
\hline & $r$ & $P$ & $r$ & $P$ & $r$ & $P$ & $r$ & $P$ & $r$ & $P$ & $r$ & $P$ & $r$ & $P$ & $r$ & $P$ & $r$ & $P$ & $r$ & $P$ \\
\hline \multicolumn{21}{|l|}{ Severe pneumonia } \\
\hline \multicolumn{21}{|l|}{ Clinical signs (days) } \\
\hline Abdominal pain & 0.30 & 0.263 & 0.06 & 0.812 & 0.03 & 0.923 & 0.05 & 0.844 & 0.38 & 0.147 & 0.32 & 0.229 & 0.07 & 0.803 & 0.78 & 0.799 & -0.37 & 0.150 & 0.04 & 0.897 \\
\hline Sickness & -0.03 & 0.906 & -0.11 & 0.676 & -0.09 & 0.742 & 0.03 & 0.902 & -0.08 & 0.759 & -0.12 & 0.661 & -0.47 & 0.093 & -0.42 & 0.153 & -0.26 & 0.306 & 0.05 & 0.842 \\
\hline Fever & 0.33 & 0.208 & 0.25 & 0.359 & 0.28 & 0.293 & 0.46 & 0.073 & 0.35 & 0.190 & -0.13 & 0.644 & -0.29 & 0.315 & -0.38 & 0.196 & -0.36 & 0.155 & 0.43 & 0.097 \\
\hline Anorexia & -0.07 & 0.795 & -0.04 & 0.874 & -0.24 & 0.374 & 0.30 & 0.263 & -0.43 & 0.097 & -0.09 & 0.731 & -0.26 & 0.377 & -0.19 & 0.530 & 0.08 & 0.766 & 0.31 & 0.238 \\
\hline Dyspnea & -0.03 & 0.905 & -0.02 & 0.945 & -0.12 & 0.654 & 0.61 & 0.012 & 0.34 & 0.196 & 0.27 & 0.308 & -0.09 & 0.761 & -0.2 & 0.523 & -0.39 & 0.126 & 0.62 & 0.010 \\
\hline Subcostal recession & 0.15 & 0.568 & 0.14 & 0.600 & -0.14 & 0.605 & 0.03 & 0.918 & 0.36 & 0.166 & -0.08 & 0.757 & 0.53 & 0.053 & -0.15 & 0.615 & -0.05 & 0.842 & 0.20 & 0.467 \\
\hline Difficult breathing & -0.10 & 0.715 & 0.09 & 0.736 & -0.21 & 0.443 & -0.05 & 0.862 & 0.09 & 0.738 & 0.23 & 0.388 & -0.12 & 0.692 & -0.17 & 0.581 & 0.18 & 0.484 & -0.01 & 0.980 \\
\hline Chest pain & -0.18 & 0.518 & 0.01 & 0.971 & -0.21 & 0.443 & 0.08 & 0.785 & -0.12 & 0.676 & -0.28 & 0.311 & -0.23 & 0.454 & -0.33 & 0.264 & -0.03 & 0.912 & -0.17 & 0.540 \\
\hline Coughing & -0.12 & 0.667 & -0.02 & 0.532 & -0.43 & 0.095 & 0.06 & 0.827 & -0.44 & 0.090 & -0.34 & 0.199 & -0.39 & 0.165 & -0.43 & 0.145 & -0.35 & 0.167 & 0.11 & 0.688 \\
\hline Vomiting & -0.31 & 0.211 & -0.24 & 0.376 & 0.16 & 0.549 & 0.58 & 0.019 & -0.12 & 0.669 & -0.08 & 0.761 & 0.22 & 0.447 & 0.28 & 0.362 & -0.02 & 0.939 & 0.55 & 0.028 \\
\hline \multicolumn{21}{|l|}{ Blood count } \\
\hline Hemoglobin & -0.06 & 0.814 & -0.18 & 0.517 & -0.16 & 0.556 & 0.18 & 0.516 & -0.12 & 0.662 & -0.62 & 0.011 & -0.03 & 0.919 & -0.25 & 0.417 & -0.35 & 0.167 & 0.18 & 0.513 \\
\hline Leukocytes & -0.34 & 0.204 & -0.25 & 0.357 & -0.11 & 0.674 & 0.51 & 0.045 & -0.08 & 0.779 & -0.24 & 0.371 & 0.4 & 0.152 & 0.31 & 0.309 & -0.28 & 0.277 & 0.52 & 0.037 \\
\hline Hemacias & -0.29 & 0.275 & -0.41 & 0.114 & -0.15 & 0.57 & 0.08 & 0.757 & -0.27 & 0.306 & -0.23 & 0.402 & -0.07 & 0.809 & -0.05 & 0.866 & -0.44 & 0.081 & 0.08 & 0.762 \\
\hline Neutrophils & -0.09 & 0.753 & -0.15 & 0.583 & -0.25 & 0.351 & 0.43 & 0.1 & -0.32 & 0.228 & -0.37 & 0.164 & -0.27 & 0.357 & -0.29 & 0.346 & -0.21 & 0.419 & 0.44 & 0.088 \\
\hline Eosinophils & -0.07 & 0.805 & -0.09 & 0.755 & -0.1 & 0.735 & -0.13 & 0.639 & 0.23 & 0.413 & -0.48 & 0.069 & -0.18 & 0.552 & -0.16 & 0.616 & 0.35 & 0.18 & -0.10 & 0.720 \\
\hline Lymphocytes & 0.26 & 0.323 & 0.37 & 0.157 & 0.32 & 0.229 & -0.33 & 0.218 & 0.45 & 0.084 & 0.46 & 0.071 & 0.32 & 0.268 & 0.31 & 0.307 & 0.4 & 0.114 & -0.33 & 0.212 \\
\hline Monocytes & 0.34 & 0.204 & 0.56 & 0.025 & 0.32 & 0.223 & -0.29 & 0.282 & 0.52 & 0.04 & 0.26 & 0.332 & 0.16 & 0.587 & 0.07 & 0.819 & 0.02 & 0.954 & -0.29 & 0.279 \\
\hline Platelets & 0.01 & 0.963 & 0.11 & 0.673 & -0.09 & 0.746 & -0.21 & 0.431 & -0.07 & 0.807 & 0.37 & 0.157 & -0.02 & 0.955 & 0.06 & 0.84 & 0.42 & 0.09 & -0.20 & 0.466 \\
\hline \multicolumn{21}{|l|}{ Non-severe Pneumonia } \\
\hline \multicolumn{21}{|l|}{ Clinical signs (days) } \\
\hline Abdominal pain & 0.91 & 0.012 & 0.89 & 0.019 & 0.81 & 0.049 & 0.35 & 0.503 & 0.81 & 0.054 & 0.40 & 0.430 & -0.38 & 0.534 & -0.56 & 0.443 & -0.38 & 0.406 & 0.34 & 0.514 \\
\hline Sickness & 0.29 & 0.579 & 0.29 & 0.287 & 0.41 & 0.418 & -0.62 & 0.19 & -0.30 & 0.569 & -0.54 & 0.273 & -0.31 & 0.612 & -0.92 & 0.077 & 0.38 & 0.401 & -0.62 & 0.185 \\
\hline Fever & 0.53 & 0.283 & 0.17 & 0.748 & 0.71 & 0.112 & 0.06 & 0.912 & 0.22 & 0.680 & -0.06 & 0.909 & 0.11 & 0.856 & 0.10 & 0.897 & -0.21 & 0.659 & -0.17 & 0.745 \\
\hline Anorexia & -0.08 & 0.887 & 0.32 & 0.538 & -0.02 & 0.975 & 0.36 & 0.479 & 0.57 & 0.237 & 0.30 & 0.561 & -0.17 & 0.781 & -0.28 & 0.722 & -0.25 & 0.591 & 0.11 & 0.832 \\
\hline Dyspnea & -0.12 & 0.822 & -0.23 & 0.658 & 0.19 & 0.722 & -0.10 & 0.852 & -0.18 & 0.739 & -0.23 & 0.666 & -0.24 & 0.693 & -0.32 & 0.681 & 0.29 & 0.535 & -0.11 & 0.837 \\
\hline Subcostal recession & - & - & - & - & - & - & - & - & - & - & - & - & - & - & - & - & - & - & - & - \\
\hline Difficult breathing & -0.13 & 0.809 & 0.32 & 0.543 & -0.12 & 0.824 & -0.19 & 0.371 & 0.18 & 0.739 & -0.17 & 0.744 & -0.38 & 0.534 & -0.38 & 0.616 & 0.33 & 0.465 & -0.20 & 0.707 \\
\hline Chest pain & - & - & - & - & - & - & - & - & - & - & - & - & -0.25 & 0.685 & - & - & 0.28 & 0.550 & - & - \\
\hline Coughing & 0.31 & 0.555 & 0.38 & 0.454 & 0.43 & 0.400 & -0.48 & 0.340 & -0.15 & 0.781 & -0.39 & 0.450 & -0.48 & 0.417 & -0.95 & 0.046 & 0.46 & 0.300 & -0.48 & 0.333 \\
\hline
\end{tabular}


Table 2 Correlation between clinical signs and blood count with cytokines serum levels of patients with severe and non-severe pneumonia (Continued)

\begin{tabular}{llllllllllllllllllllllllllllllll}
\hline $\begin{array}{l}\text { Vomiting } \\
\text { Blood count }\end{array}$ & -0.09 & 0.884 & 0.13 & 0.839 & -0.21 & 0.731 & $\mathbf{1 . 0 0}$ & $<\mathbf{0 . 0 0 1}$ & 0.73 & 0.164 & $\mathbf{0 . 9 9}$ & $\mathbf{0 . 0 0 1}$ & - & - & - & - & -0.58 & 0.229 & $\mathbf{1 . 0 0}$ & $<\mathbf{0 . 0 0 1}$ \\
Hemoglobin & 0.62 & 0.189 & 0.24 & 0.641 & 0.54 & 0.27 & 0.3 & 0.57 & 0.42 & 0.402 & 0.23 & 0.66 & $\mathbf{0 . 9 7}$ & $\mathbf{0 . 0 0 6}$ & $\mathbf{0 . 9 7}$ & $\mathbf{0 . 0 3 1}$ & $\mathbf{- 0 . 8 2}$ & $\mathbf{0 . 0 2 4}$ & 0.29 & 0.575 \\
Leukocytes & -0.55 & 0.256 & -0.35 & 0.496 & -0.68 & 0.138 & 0.49 & 0.328 & 0.16 & 0.765 & 0.44 & 0.387 & 0.54 & 0.348 & 0.72 & 0.282 & -0.48 & 0.273 & 0.49 & 0.319 & \\
Hemacias & -0.45 & 0.371 & -0.56 & 0.251 & -0.65 & 0.166 & 0.29 & 0.571 & -0.23 & 0.666 & 0.31 & 0.552 & 0.71 & 0.178 & 0.74 & 0.256 & -0.49 & 0.268 & 0.31 & 0.551 \\
Neutrophils & -0.64 & 0.172 & -0.21 & 0.688 & -0.76 & 0.078 & 0.49 & 0.321 & 0.2 & 0.699 & 0.5 & 0.311 & -0.01 & 0.987 & 0.1 & 0.9 & -0.18 & 0.707 & 0.50 & 0.313 \\
Eosinophils & 0.49 & 0.325 & 0.19 & 0.714 & 0.71 & 0.113 & -0.38 & 0.455 & -0.13 & 0.813 & -0.43 & 0.399 & -0.31 & 0.61 & -0.4 & 0.597 & 0.33 & 0.467 & -0.39 & 0.440 \\
Lymphocytes & 0.73 & 0.097 & 0.47 & 0.346 & $\mathbf{0 . 8 7}$ & $\mathbf{0 . 0 2 5}$ & -0.48 & 0.333 & -0.01 & 0.986 & -0.47 & 0.345 & -0.45 & 0.443 & -0.62 & 0.379 & 0.3 & 0.516 & -0.49 & 0.320 \\
Monocytes & 0.08 & 0.888 & -0.24 & 0.646 & 0.36 & 0.483 & -0.44 & 0.386 & -0.39 & 0.448 & -0.56 & 0.247 & 0.02 & 0.98 & -0.09 & 0.913 & 0.23 & 0.623 & -0.45 & 0.374 \\
Platelets & -0.42 & 0.411 & -0.4 & 0.438 & -0.34 & 0.506 & -0.48 & 0.338 & -0.71 & 0.115 & -0.4 & 0.43 & -0.39 & 0.519 & -0.56 & 0.439 & 0.7 & 0.08 & -0.47 & 0.346 \\
\hline
\end{tabular}

Note: $r$, Person correlation. $P$-value $<0.05$ was considered significant, significant values are shown in bold 

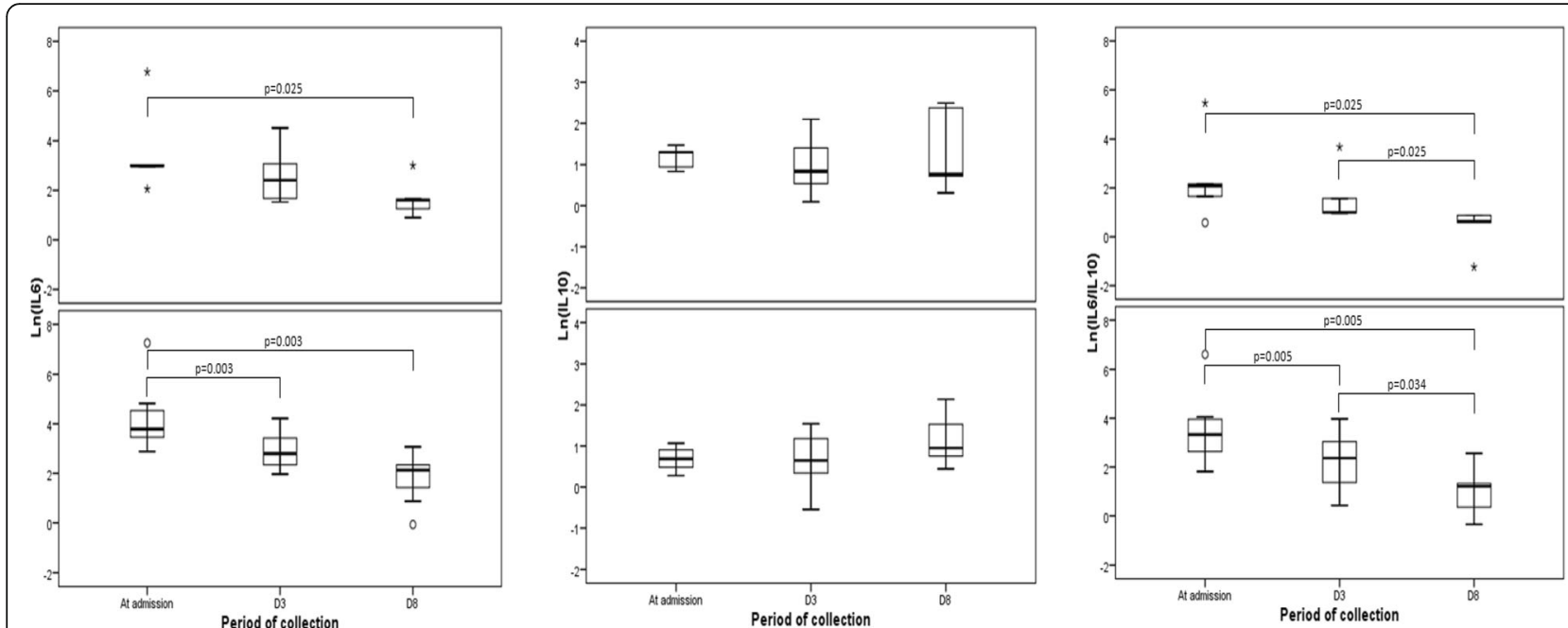

Fig. 1 Balance between IL-6 and IL-10 during recovery from pneumonia of different severity. Seric levels of IL-6 (left) and IL-10 (middle) cytokines, and the ratio of seric levels of IL-6:L-10 (right) in group of children diagnosed with non-severe pneumonia (top) or severe pneumonia (bottom). IL-6 levels, in non-severe pneumonia, at admission $\times$ D8 $(P=0.025)$; IL-6 levels, in severe pneumonia, at admission $\times$ D3 $(P=0.003)$ and at admission x D8 ( $P=0.003)$; Ratio IL-6:IL-10, in non-severe pneumonia, at admission and D3 $(P=0.025)$ and D3 x D8 $(P=0.025)$; Ratio IL-6:IL-10, in severe pneumonia, at admission x D3 $(P=0.005)$; at admission and D8 $(P=0.005)$ and D3 $\times$ D8 $(P=0.034)$

should be considered. First, a possible delay in diagnosis and treatment in the residence's town may have contribute to an hemodynamic instability and ICU requirement at admission in the hospital; secondly, the innate immunity of young children may be compromised by less bactericidal activity and inflammatory response by immature neutrophils, and poor cytokine response by macrophages and monocytes [40].

Inflammation biomarkers, as cytokines, may be useful in determining the magnitude of the inflammatory response and lung injury in children with pneumonia. However, studies that tried to associate clinical severity in adult $[18,21,41]$ and children [42] patients with respiratory diseases and serum levels of cytokines had controversial results. In the present study, the cytokines, TNF, IL-1 $\beta$, IL-6, IL-8, IL-12p70, IFN- $\gamma$, IL-17A, IL-10 and IL-5, were detected in the serum of children with pneumonia and severe pneumonia at hospital admission, and IL-6 was the only cytokine associated with disease severity.

Table 3 Interleukin (IL)-6 and the IL-6:IL-10 serum levels ratio as marker of pneumonia severity and recovery

\begin{tabular}{|c|c|c|c|c|c|c|c|c|c|}
\hline \multicolumn{5}{|l|}{ IL-6 } & \multicolumn{5}{|l|}{ IL-6/IL-10 } \\
\hline Time-points & $\begin{array}{l}\text { Severe } \\
\text { pneumonia }\end{array}$ & $\begin{array}{l}\text { Non-severe } \\
\text { pneumonia }\end{array}$ & & $\mathrm{Cl} 95 \%$ & Time-points & Severe pneumonia & $\begin{array}{l}\text { Non-severe } \\
\text { pneumonia }\end{array}$ & & $\mathrm{Cl} 95 \%$ \\
\hline At admission & & & Sen $=76.5$ & $49.8-92.2$ & At admission & & & Sen $=76.5$ & $49.8-92.2$ \\
\hline$>21.1$ & 13 & 1 & Spec $=83.3$ & $36.5-99.1$ & $>9.61$ & 13 & 1 & Spec $=83.3$ & $36.5-99.1$ \\
\hline \multirow[t]{2}{*}{$\leq 21.1$} & 4 & 5 & $P P V=92.9$ & $64.2-99.6$ & $\leq 9.61$ & 4 & 5 & $P P V=92.9$ & $64.2-99.6$ \\
\hline & & & $N P V=55.6$ & $22.7-84.7$ & & & & $N P V=55.6$ & $22.7-84.7$ \\
\hline D3 & & & Sen $=58.3$ & $28.6-83.5$ & D3 & & & Sen $=72.7$ & $39.3-92.7$ \\
\hline$>11.28$ & 7 & 2 & Spec $=60.0$ & $17.0-92.7$ & $>5.00$ & 8 & 1 & Spec $=80.0$ & $29.9-98.9$ \\
\hline \multirow[t]{2}{*}{$\leq 11.28$} & 5 & 3 & $P P V=77.8$ & $40.2-96.1$ & $\leq 5.00$ & 3 & 4 & $P P V=88.9$ & $50.7-99.4$ \\
\hline & & & $N P V=37.5$ & $10.2-74.1$ & & & & NPV $=57.1$ & $20.2-88.2$ \\
\hline D8 & & & & & D8 & & & & \\
\hline$>5.07$ & 8 & 2 & Sen $=66.7$ & $35.4-88.7$ & $>2.40$ & 7 & 1 & $\operatorname{Sen}=63.6$ & $31.6-87.6$ \\
\hline \multirow[t]{3}{*}{$\leq 5.07$} & 4 & 3 & Spec $=60.0$ & $17.0-92.7$ & $\leq 2.40$ & 4 & 4 & Spec $=80.0$ & $29.9-98.9$ \\
\hline & & & $P P V=80.0$ & $44.2-96.5$ & & & & $P P V=87.5$ & $46.7-99.3$ \\
\hline & & & $\mathrm{NPV}=42.9$ & $11.8-79.8$ & & & & $N P V=50.0$ & $17.4-82.6$ \\
\hline
\end{tabular}

Note: D0 day of admission, D3 third day and D8 - eighth day of hospitalization, Sen sensitivity, Spec specificity, PPV positive predictive value, NPV negative predictive value, $\mathrm{Cl}$ confidence interval 
At admission (D0), we observed high serum levels of cytokines, predominantly pro-inflammatory including TNF, IL-1 $\beta$, IL- 6 and IL-8, which are involved in the innate immune response and in the immune response development $[22,43,44]$. In patients with severe pneumonia, the IL-6 levels were positively associated with high levels of leukocytes while the levels of TNF and IL-1 $\beta$ were associated with monocytes, possibly related to the recruitment and differentiation of macrophages at the site of inflammation. According to Bauer et al., TNF and IL-1 $\beta$ are associated with lung injury in acute respiratory distress syndrome and in severe pneumonia, although in a smaller magnitude [21]. A study reported by Kolsuz et al. on inflammatory cytokines (TNF, IL-1 $\beta$, IL-6 and IL-8) levels in bronchoalveolar material after $24 \mathrm{~h}$ of admission for adult patients with pneumonia showed IL- 6 as the most important cytokine in the determination of the disease severity in patients with systemic inflammatory response syndrome [45]. In other study, Antunes et al. reported high levels of IL-6, TNF- $\alpha$, IL-10 and IL-1 $\beta$ detected in the majority of the 24 patients with CAP at admission, but the cytokines levels decreased significantly on days three and five of hospitalization, and IL-6 was the only cytokine associated with the disease severity, as ours findings [9].

High levels of IL-6 were observed in both studied groups at admission, highlighting the importance of this cytokine in the pulmonary inflammatory process regardless the disease severity [9]. Although IL-6 levels have been correlated with vomiting, which is a clinical manifestation of disease severity, other causes, such as the use of medications and inflammatory systemic process, may implicate in the etiology of vomiting and abdominal pain [34, 37]. IL-6 was also positively correlated with dyspnea, which may represent, under the point of view of the pathophysiology, the intensity of the lung's inflammatory process, which is associated with respiratory disease severity.

An important anti-inflammatory cytokine in pneumonia is IL-10. In the present study, IL-10 levels were higher in the group of patients with pneumonia than in patients with severe pneumonia in admission $(1.99 \times 3.68 \mathrm{pg} / \mathrm{mL}$; $P=0.074$ ). Although the statistical differences were not significant what may be related to the small number of samples, the increased levels of IL-10 in patients with pneumonia was associated with high levels of lymphocytes, suggesting that IL-10 is an important modulatory factor of the inflammatory response of acute pneumonia $[6,24]$.

\section{Conclusion}

In conclusion, the controversial results of some studies regarding the correlation of IL-6 serum levels and pneumonia severity may be produced by the choice of disease time point analysed and the balance between IL- 6 and IL-10 serum levels. Our findings showed the ratio between the serum levels of IL- 6 and IL-10 is an important criteria for severity definition at hospital admission, allowing to screening at the emergency room who is at risk of evolving into complication, and evaluation of recovery capacity. Specially in the third day of antibiotic therapy, when the patients' clinical condition is usually evaluated for possible changing in the treatment protocol, an IL-6:IL-10 ratio higher than 5.0 may predict that $89 \%$ of positive cases have still severe pneumonia, indicating that changes in the treatment protocol might be needed. In future, the determination of IL-6 and IL-10 serum levels may be offered by routine laboratory as one more marker to evaluate the severity of infection disease at the diagnosis.

\section{Additional files}

Additional file 1: Table S1. Clinical features of children with severe pneumonia and non-severe pneumonia. The differences between the clinic of children with severe and non-severe pneumonia included anorexia, wheezing, respiratory difficulty, subcostal recession, $\mathrm{O} 2$ saturation, but not respiratory or heart frequencies, fever or caughing; the duration of fever and hospitalization was longer in severe pneumonia. (DOC $75 \mathrm{~kb}$ )

Additional file 2: Table S2. Temporal evolution of clinical signs of patients according the pneumonia severity. Comparison of clinical signs inside the severe pneumonia group (Intragroup analysis) between different sampling times showed that changes in corporal temperature, $\mathrm{O}_{2}$ saturation and respiratory frequency were observed only in the eighth day (D8) of hospitalization; while in non-severe pneumonia group changes on corporal temperature and respiratory frequency were observed in the third day (D3) of hospitalization, confirming the delay recovery of severe pneumonia. Intergroup analysis corresponded to the comparison of clinical signs between severe and non-severe pneumonia groups in each time-point and showed significative differences in $\mathrm{O}_{2}$ saturation on D3 and D8 in relation to diagnosis (D0), as expected since it definies disease severity; differences in corporal temperature on D3 and respiratory frequency on D3 and D8 showed to be helpful to distinguish cases with unfavored evolution, since non-severe pneumonia recovery faster. (DOC 62 kb)

\section{Abbreviations}

CAP: Community acquired pneumonia; CRP: C-reactive protein; ICU: Intensive care unit; IFNY: Interferon gamma; IL: Interleukin; TNF: Tumor necrosis factor; WBC: White blood cell; WHO: World Health Organization

\section{Acknowledgments}

We thank the under graduated student in Medicina Talitha Coelho Moraes Guerra, Luciana de Holanda Lima Dornelas Câmara and Juliana Dias Pereira Gomes de Mattos for their care and data collection at the patient bedside.

Funding

This study was funded by Grant: PRONEM/FACEPE \#1279-4.01/10.

\section{Availability of data and material}

The datasets used and analysed during the current study are available from the corresponding author on reasonable request.

\section{Authors' contributions}

RCCMB conceptualized and designed the study, developed the questionnaire, coordinated and supervised the data collection, analyzed the data, drafted the manuscript and approved the final manuscript as submitted. LCT performed the flow cytometry and ELISA analyses, drafted the initial manuscript and approved the final manuscript as submitted. CFL developed the questionnaire and analyzed the data, drafted the initial manuscript and approved the final manuscript as submitted. GAPS, JBCLS and NLS conceptualized and designed the study, analyzed the data, revised the manuscript and approved the final 
manuscript as submitted. All authors approved the final manuscript as submitted, and agreed to be accountable for all aspects of the work.

\section{Competing interests}

The authors have no conflict of interest to disclose. The authors have indicated they have no financial relationships relevant to this article to disclose.

\section{Consent for publication}

Not applicable.

\section{Ethics approval and consent to participate}

The present study was approved by the Research Ethics Committees of the IMIP hospital (protocol 2886) and the Hospital da Restauração (protocol CAAE-0014.0.102.000-11). A clearance letter was also issued by the Health Secretary of the state of Pernambuco authorizing the research implementation in the Hospital Helena Moura. Data collection was performed after obtaining written Informed Consent signed by the children's legal guardian.

\section{Author details}

'Institute of Integral Medicine Professor Fernando, Figueira (IMIP), Pediatrics, Rua Dona Benvinda de Farias 159, apt 1101, Boa Viagem, Recife, Pernambuco, Brazil. ${ }^{2}$ Institute of Integral Medicine Professor Fernando Figueira (IMIP), Oncology and Aggeu Magalhães Research Center, Fiocruz-PE, Immunology, Recife, Brazil. ${ }^{3}$ Institute of Integral Medicine Professor Fernando, Figueira, IMIP, Translational Medicine Laboratory, Recife, Brazil. ${ }^{4}$ Aggeu Magalhães Research Center, Fiocruz-PE, Public Health, Recife, Brazil. ${ }^{5}$ Federal University of Pernambuco, Pediatrics, Recife, Brazil.

\section{Received: 10 June 2016 Accepted: 17 November 2016} Published online: 01 December 2016

\section{References}

1. Nair H, Simões EAF, Rudan I, et al. Global and regional burden of hospital admissions for severe acute lower respiratory infections in young children in 2010: a systematic analysis. Lancet. 2013;381(9875):1380-90.

2. Jackson S, Mathews KH, Pulanić D, et al. Risk factors for severe acute lower respiratory infections in children - a systematic review and meta-analysis. Croat Med J. 2013;54(2):110-21

3. Management DI, Society D. Diretrizes brasileiras em pneumonia adquirida na comunidade em pediatria - 2007. Pneumonias comunitárias. 2007; 33(1):31-50.

4. World Health Organization. Pocket book of hospital care for children: guidelines for the management of common childhood illnesses. 2nd ed. Geneva: World Health Organization; 2013

5. Tiewsoh K, Lodha R, Pandey RM, Broor S, Kalaivani M, Kabra SK. Factors determining the outcome of children hospitalized with severe pneumonia. BMC Pediatr. 2009:9:15. doi:10.1186/1471-2431-9-15.

6. Gallagher PM, Lowe G, Fitzgerald T, et al. Association of IL-10 polymorphism with severity of illness in community acquired pneumonia. Thorax. 2003; 58(2):154-6

7. Endeman H, Rijkers GT, Grutters JC, Biesma DH. Systemic cytokine response in patients with community-acquired pneumonia. Eur Respir J. 2011; 37(6):1431-8.

8. Solé-Violán J, de Castro FV, García-Laorden Ml, et al. Genetic variability in the severity and outcome of community-acquired pneumonia. Respir Med. 2010;104(3):440-7.

9. Antunes G, Evans SA, Lordan JL, Frew AJ. Systemic cytokine levels in community-acquired pneumonia and their association with disease severity. Eur Respir J. 2002;20:990-5

10. Ekiz C, Agaoglu L, Karakas Z, Gurel N, Yalcin I. The effect of iron deficiency anemia on the function of the immune system. Hematol J. 2005;5(7):579-8.

11. Hussain SQ, Ashraf M, Wani JG, Ahmed J. Low Hemoglobin Level a Risk Factor for Acute Lower Respiratory Tract Infections (ALRTI) in Children. J Clin Diagn Res. 2014;8(4):C01-3. doi:10.7860/JCDR/2014/8387.4268. Epub 2014 Apr 15.

12. Carvalho AGC, de Lira PIC, Barros MFA, Aléssio MLM, Lima MC, Carbonneau MA, Berger J, Léger CL. Diagnosis of iron deficiency anemia in children of Northeast Brazil. Rev Saude Publica. 2010;44(3):513-9.

13. Chisti MJ, Tebruegge M, La Vincente S, Graham SM, Duke T. Pneumonia in severely malnourished children in developing countries - mortality risk, aetiology and validity of WHO clinical signs: a systematic review. Trop Med Int Health. 2009:14(10):1173-89.

14. Virkki R, Juven T, Rikalainen H, Svedström E, Mertsola J, Ruuskanen O. Differentiation of bacterial and viral pneumonia in children. Thorax. 2002; 57(5):438-41.

15. Thomas MF, Spencer DA. Management and complications of pneumonia. Paediatr Child Health (Oxford). 2011;21(5):207-12.

16. Lynch $T$, Bialy L, Kellner JD, et al. A systematic review on the diagnosis of pediatric bacterial pneumonia: when gold is bronze. PLoS One. 2010; 5(8):e11989.

17. Don M, Valent F, Korppi M, Canciani M. Differentiation of bacterial and viral community-acquired pneumonia in children. Pediatr Int. 2009;51(1):91-6.

18. Abdellaoui A, Al-Khaffaf H. C-reactive protein (CRP) as a marker in peripheral vascular disease. Eur J Vasc Endovasc Surg. 2007;34(1):18-22.

19. Park WY, Goodman RB, Steinberg KP, Ruzinski JT, Radella 2nd F, Park DR, Pugin J, Skerrett SJ, Hudson LD, Martin TR. Cytokine Balance in the Lungs of Patients with Acute Respiratory Distress Syndrome. Am J Respir Crit Care Med. 2001;164(10 Pt 1):1896-903.

20. Suzuki T, Chow C-W, Downey GP. Role of innate immune cells and their products in lung immunopathology. Int J Biochem Cell Biol. 2008;40(6-7):1348-61.

21. Bauer T, Montón C, Torres A, et al. Comparison of systemic cytokine levels in patients with acute respiratory distress syndrome, severe pneumonia, and controls. Thorax. 2000:55:46-52.

22. Medzhitov R, Janeway Jr CA. Innate immune recognition and control of adaptive immune responses. Semin Immunol. 1998;10(5):351-3.

23. Deng JC, Standiford TJ. The systemic response to lung infection. Clin Chest Med. 2005;26(1):1-9. doi:10.1016/j.ccm.2004.10.009.

24. Waterer GW. Airway defense mechanisms. Clin Chest Med. 2012;33(2):199-209. doi:10.1016/j.ccm.2012.03.003.

25. Heinrich PC, Behrmann I, Haan S, Hermanns HM, Müller-Newen G, Schape F. Principles of interleukin (IL)-6-type cytokine signalling and its regulation. Biochem J. 2003:374(Pt 1):1-20.

26. Menendez R, Lorenzo MJ, Moret I, Sarria B, Cases E, Molina JM, Gimeno A. Cytokine Profile And Antibiotic Therapy In Community-Acquired Pneumonia A25. The Changings face of pneumonia and its management. 2013. p. A1146.

27. Brasil. Ministério da Saúde. Secretaria de Atenção à Saúde. Ministério da Saúde. Secretaria de Atenção à Saúde. Departamento de Atenção Básica. Brasília: Ministério da Saúde: 2012.

28. Rudan I, Brien KLO, Qazi S, Walker CLF, Black RE, Campbell H. Child Health Epidemiology Reference Group (CHERG). Epidemiology and etiology of childhood pneumonia in 2010: estimates of incidence, severe morbidity, mortality, underlying risk factors and causative pathogens for 192 countries. J Glob Health. 2013;3(1):010401. doi:10.7189/jogh.03.010401.

29. Gentile A, Bardach A, Ciapponi A, et al. Epidemiology of communityacquired pneumonia in children of Latin America and the Caribbean: a systematic review and meta-analysis. Int J Infect Dis. 2012;16(1):e5-15.

30. Izadnegahdar R, Cohen AL, Klugman KP, Qazi SA. Childhood pneumonia in developing countries. Lancet Respir. 2013;1(7):574-84.

31. Cevey-Macherel M, Galetto-Lacour A, Gervaix A, et al. Etiology of community- acquired pneumonia in hospitalized children based on WHO clinical guidelines. Eur J Pediatr. 2009;168(12):1429-36.

32. Murphy CG, van de Pol AC, Harper MB, Bachur RG. Clinical predictors of occult pneumonia in the febrile child. Acad Emerg Med. 2007;14(3):243-9. doi:10.1197/j.aem.2006.08.022.

33. de Britto MC, da Conceicao Silvestre SM. do Carmo Menezes Duarte M, Matos Bezerra PG. Clinical profile of pleural empyema and associated factors with prolonged hospitalization in paediatric tertiary centre in Angola, Luanda. Trop Doct. 2008:38(2):118-20.

34. Tsalkidis A, Gardikis S, Cassimos D, et al. Acute abdomen in children due to extra-abdominal causes. Pediatr Int. 2008:50(3):315-8.

35. Clark JE, Hammal D, Hampton F, Spencer D, Parker L. Epidemiology of community-acquired pneumonia in children seen in hospital. Epidemiol Infect. 2007;135(2):262-9

36. Harris $M$, Coote $N$, Fletcher $P$, et al. British Thoracic Society guidelines for the management of community acquired pneumonia in children: update 2011. Thorax. 2011;66 Suppl 2:ii1-23. doi:10.1136/thoraxinl-2011-200598.

37. Moustaki M, Zeis PM, Katsikari M, et al. Mesenteric lymphadenopathy as a cause of abdominal pain in children with lobar or segmental pneumonia. Pediatr Pulmonol. 2003;35(4):269-73.

38. Brabin BJ, Premji Z, Verhoeff F. An Analysis of Anemia and Child Mortality. J Nutr. 2001;131(2S-2):636S-45S. discussion 646S-648S. 
39. Pinto KDBPC, Maggi RRS, Alves GB. Análise de risco sócio-ambiental para comprometimento pleural na pneumonia grave em crianças menores de 5 anos. Rev Panam Salud Publica/Pan Am J Public Health. 2004;15(2):104-9.

40. Simon AK, Hollander GA, McMichael A. Evolution of the immune system in humans from infancy to old age. Proc R Soc B. 2015;282:20143085.

41. Sprong T, van der Ven-Jongekrijg J, Neeleman C, van der Meer JW, van Deuren M. Influence of innate cytokine production capacity on clinical manifestation and severity of pediatric meningococcal disease. Crit Care Med. 2009;37(10):2812-8. doi:10.1097/CCM.0b013e3181ab851c.

42. Michelow IC, Katz K, Mccracken GH, Hardy RD. Systemic Cytokine Profile in Children With Community-Acquired Pneumonia. Pediatr Pulmonol. 2007; 42(7):640-5

43. Knapp S, Hareng L, Rijneveld AW, et al. Activation of neutrophils and inhibition of the proinflammatory cytokine response by endogenous granulocyte colony-stimulating factor in murine pneumococcal pneumonia. J Infect Dis. 2004;189(8):1506-15.

44. Alcón A, Fàbregas N, Torres A. Pathophysiology of pneumonia. Clin Chest Med. 2005;26(1):39-46.

45. Kolsuz M, Erginel S, Alatas O, et al. Acute Phase Reactants and Cytokine Levels in Unilateral Community-Acquired Pneumonia. Respiration. 2003; 70(6):615-22.

\section{Submit your next manuscript to BioMed Central} and we will help you at every step:

- We accept pre-submission inquiries

- Our selector tool helps you to find the most relevant journal

- We provide round the clock customer support

- Convenient online submission

- Thorough peer review

- Inclusion in PubMed and all major indexing services

- Maximum visibility for your research

Submit your manuscript at www.biomedcentral.com/submit 